\title{
Whole-slide image analysis of the tumor microenvironment identifies low B-cell content as a predictor of adverse outcome in patients with advanced-stage classical Hodgkin lymphoma treated with BEACOPP
}

Haematologica 2021

Volume 106(6):1684-1692

\section{Correspondence:}

RON D. JACHIMOWICZ

ron.jachimowicz@uk-koeln.de

WOLFRAM KLAPPER

wklapper@path.uni-kiel.de

Received: December 12, 2019.

Accepted: April 29, 2020.

Pre-published: May 7, 2020.

https://doi.org/10.3324/haematol.2019.243287

(C)2021 Ferrata Storti Foundation

Material published in Haematologica is covered by copyright. All rights are reserved to the Ferrata Storti Foundation. Use of published material is allowed under the following terms and conditions:

https://creativecommons.org/licenses/by-nc/4.0/legalcode. Copies of published material are allowed for personal or internal use. Sharing published material for non-commercial purposes is subject to the following conditions:

https://creativecommons. org//icenses/by-nc/4.0/legalcode, sect. 3. Reproducing and sharing published material for commercial purposes is not allowed without permission in writing from the publisher.
Ron Daniel Jachimowicz,,$^{1,2,3^{*}}$ Luise Pieper, ${ }^{4 *}$ Sarah Reinke, ${ }^{4, *}$ Artur Gontarewicz, ${ }^{4, *}$ Annette Plütschow, ${ }^{1}$ Heinz Haverkamp, ${ }^{1}$ Leonie Frauenfeld, ${ }^{5}$ Falko Fend, ${ }^{5}$ Mathis Overkamp, ${ }^{5}$ Franziska Jochims, ${ }^{4}$ Christoph Thorns, ${ }^{6}$ Martin Leo Hansmann, ${ }^{7}$ Peter Möller, ${ }^{8}$ Andreas Rosenwald, ${ }^{9}$ Harald Stein, ${ }^{10}$ Hans Christian Reinhardt, $, 2,31,12$ Peter Borchmann,,$^{1,3}$ Bastian von Tresckow, ${ }^{1,3}$ Andreas Engert ${ }^{1,3}$ and Wolfram Klapper $^{4}$

${ }^{1}$ University of Cologne, Faculty of Medicine and University Hospital Cologne, Department I of Internal Medicine, Cologne; German Hodgkin Study Group, Cologne; ${ }^{2}$ Else Kröner Forschungskolleg Clonal Evolution in Cancer, University Hospital Cologne, Cologne; ${ }^{3}$ University of Cologne, Faculty of Medicine and University Hospital Cologne, Department I of Internal Medicine, Center for Integrated Oncology Aachen-Bonn-Cologne-Düsseldorf, Cologne; ${ }^{4}$ Department of Pathology, Hematopathology Section, University Hospital Schleswig-Holstein, Christian-Albrechts-University, Kiel; ${ }^{518}$ Department of Pathology, University of Tübingen, Tübingen; 'Department of Pathology, University Hospital SchleswigHolstein, University of Lübeck, Lübeck; ' ${ }^{7}$ Department of Pathology, University Hospital Frankfurt, Frankfurt; ${ }^{2}$ Department of Pathology, University Hospital Ulm, Ulm; ' Institute of Pathology, University of Würzburg, and Comprehensive Cancer Center Mainfranken, Würzburg; ${ }^{10}$ Pathodiagnostik, Berlin; ${ }^{11}$ Cologne Excellence Cluster on Cellular Stress Response in Aging-Associated Diseases (CECAD), University of Cologne, Cologne and ${ }^{12}$ Center for Molecular Medicine Cologne (CMMC), University of Cologne, Cologne, Germany

${ }^{*} R D J, L P, S R$ and $A G$ contributed equally as co-first authors.

\section{ABSTRACT}

A subset of patients with advanced-stage classical Hodgkin lymphoma (cHL) relapse or progress following standard treatment. Given their dismal prognosis, identifying this group of patients upfront represents an important medical need. While prior research has identified characteristics of the tumor microenvironment, which are associated with cHL outcomes, biomarkers that are developed and validated in this high-risk group are still lacking. Here, we applied wholeslide image analysis (WSI), a quantitative, large-scale assessment of tumor composition that utilizes conventional histopathology slides. We conducted WSI on pre-treatment biopsies from 340 patients with advanced-stage cHL enrolled in the HD12 and HD15 trials of the German Hodgkin Study Group (GHSG), and tested our results in a validation cohort of 147 advanced-stage cHL patients within the GHSG HD18 trial. All patients were treated with BEACOPP-based regimens. By quantifying T cells, B cells, Hodgkin and Reed-Sternberg cells and macrophages with WSI, $80 \%$ of all cells in the tumor tissue were identified. Crucially, low B-cell count was associated with significantly reduced progression-free survival and overall survival, while the content of T cells, macrophages and Hodgkin and Reed-Sternberg cells was not associated with the risk of progression or relapse in the study cohort. We further validated low Bcell content as a prognostic factor for progression-free survival and overall survival in the validation cohort and demonstrated the good interobserver agreement of WSI. WSI may represent a key tool for risk stratification of advanced-stage cHL and can easily be added to the standard diagnostic histopathology work-up. 


\section{Introduction}

Classical Hodgkin lymphoma (cHL) is a B-cell-derived lymphoid malignancy, affecting 2.5-3/100,000 people per year. Depending on the clinical stage and associated risk factors, first-line treatment consists of poly-chemotherapy with or without consolidating radiotherapy, and results in long-term tumor control in 70-80\% and overall survival of $80-90 \%$ of patients. ${ }^{1,2}$ Crucially, all patients with advanced-stage cHL currently continue to receive aggressive poly-chemotherapy within the German Hodgkin Study Group (GHSG) clinical trials. This treatment regimen is common practice because, to date, there is no reliable tool to distinguish, a priori, the subset of patients at high risk of relapse or progressive disease from those with lower risk. As a result, two groups of patients may be underserved by current treatment approaches: those who could be treated with less toxic treatment regimens; and those who are likely to relapse or progress after standard chemotherapy, as their prognosis is often very poor with less than $50 \%$ becoming long-term survivors. ${ }^{3}$ In contrast to non-Hodgkin lymphoma, clinical risk indices for $\mathrm{cHL}$ such as the International Prognostic Score (IPS) have not been successfully applied in treatment decision-making within the subgroup of patients with advanced stage cHL. ${ }^{4}$

Similarly, the commonly defined histological subtypes of cHL lack prognostic significance under the currently used standard treatment protocols. Four microenvironmental patterns reflect the basis of the histological subtypes in the current World Health Organization classification, namely nodular sclerosis, mixed cellularity, lymphocyte-rich and lymphocyte-depleted. In recent research, however, the tumor microenvironment of $\mathrm{cHL}$ has attracted interest as a predictor of disease outcome. ${ }^{5} \mathrm{cHL}$ is characterized by the presence of neoplastic Hodgkin and Reed-Sternberg cells (HRSC) that constitute only a minority of cells within the affected lymph nodes. The tumor is mainly composed of non-neoplastic stromal and immune cells, which form a tumor microenvironment around HRSC. Depending on the cHL subtype, the cellular part of the tumor microenvironment is made up of variable proportions of macrophages, neutrophils, eosinophils, T cells, $\mathrm{B}$ cells, fibroblasts and plasma cells. The neoplastic HRSC is dependent on both endogenous- and exogenous signals, the latter stemming from crosstalk with the microenvironment, e.g., by the interaction between macrophages and tumor cells. ${ }^{6}$

The prognostic value of HRSC CD20 expression is controversial. ${ }^{7-10}$ Numerous studies utilizing quantitative assessment of mRNA or immunohistochemistry have suggested an adverse effect of increased macrophage infiltration $^{11-15}$ and a favorable effect for markers of normal B cells in the tumor microenvironment of cHL. ${ }^{16-18}$ However, the immunohistochemistry-based approaches used to assess the microenvironmental composition and the prognostic impact suffer from limitations hampering their integration into routine diagnostic procedures and clinical decisionmaking for cHL. Unlike gene expression analysis, which usually analyzes bulk tumor tissue, including the nonmalignant microenvironment, classical immunohistochemical studies analyze a limited size of tumor regions and may be biased by intratumoral heterogeneity and observer-dependent selection of the areas analyzed. Moreover, validation of microenvironmental biomarkers has rarely been performed in large clinical trial cohorts. To overcome these limitations and to test the prognostic role of the tumor microenvironment in advanced-stage cHL, we performed whole-slide-image analysis (WSI) of T cells (CD3), B cells (CD20), HRSC (CD30) and macrophages (CD68) in a study cohort and an independent validation cohort consisting of hundreds of patients treated within trials of the German Hodgkin Study Group (GHSG).

\section{Methods}

\section{Study design and patients' samples}

Between January 1999 and July 2017, 5,801 adult patients with primarily diagnosed and histologically confirmed advanced-stage cHL were randomized to receive first-line treatment with the BEACOPP regimen, containing bleomycin, etoposide, doxorubicin, cyclophosphamide, vincristine, procarbazine, and prednisone in standard or escalated (eBEACOPP) doses within the randomized GHSG clinical trials HD12, HD15, and HD18. ${ }^{19-21}$ Our cohort comprised trial patients with (cases) and without (controls) progression or relapse at the ratio of 1:2.

Inclusion criteria for the case-control cohort were documentation of the reference histology result and reference center and availability of a formalin-fixed paraffin-embedded lymph-node specimen obtained at first diagnosis as well as complete documentation of the presence or absence of prognostic factors. Interobserver agreement of WSI was tested using randomly selected WSI slides analyzed by a second observer and a randomly selected cohort stained and analyzed in a second center (Online Supplementary Methods).

The WSI findings of the study cohort of patients $(n=340)$ from HD12 and HD15 (Figure 1A) were matched with clinical data and analyzed for prognosis. The performance was subsequently tested using an independent validation cohort comprising HD18 patients $(\mathrm{n}=147)$ (Figure 1B). Median follow-up times were 66 months (95\% confidence interval [95\% CI]: 63-71) in the study cohort and 62 months (95\% CI: $57-65)$ in the validation cohort.

Table 1 summarizes the clinical characteristics of the study and validation cohorts in relation to all randomized HD12 and HD15 patients or all randomized HD18 patients, respectively. Patients included in the study cohort were slightly younger compared to all patients from the HD12 and HD15 randomized cohorts (median age: 31 years [range, 24-40] vs. 33 years [25-42], respectively; $P=0.0093)$. There were no differences in prognostically relevant laboratory parameters nor the IPS scores; however, more patients in the study cohort had $\geq 3$ nodal areas affected and stage IV disease at diagnosis (Table 1A). The validation cohort did not differ from the HD18 randomized cohort regarding demographic and prognostic factors (Table 1B). The study was conducted in accordance with the recommendations of the ethics board of the Medical Faculty, University of Kiel.

\section{Whole-slide image analysis}

For each patient, the whole available tissue specimen/block was cut and subsequent slides were stained in the Department of Pathology at Kiel University for CD3 (clone SP7, Waltham, MA, USA), CD20 (clone L26, Dako, Glostrup, Denmark), CD30 (clone BerH2, maintained at the Department of Pathology, Kiel, Germany) and CD68 (clone PG-M1, Dako, Glostrup, Denmark), using a Leica-Bond-Max stainer (Leica Microsystems, Wetzlar, Germany). The slides were scanned (Hamamatsu Nanozoomer, Hamamatsu Photonics, Ammersee, Germany) and the resulting images were processed by TissueStudio 64, according to the manufacturer's recommendations (Definiens AG, Munich, Germany). The area ranged between 4-455 $\mathrm{mm}^{2}$ (mean: $133.81 \mathrm{~mm}^{2}$, standard 


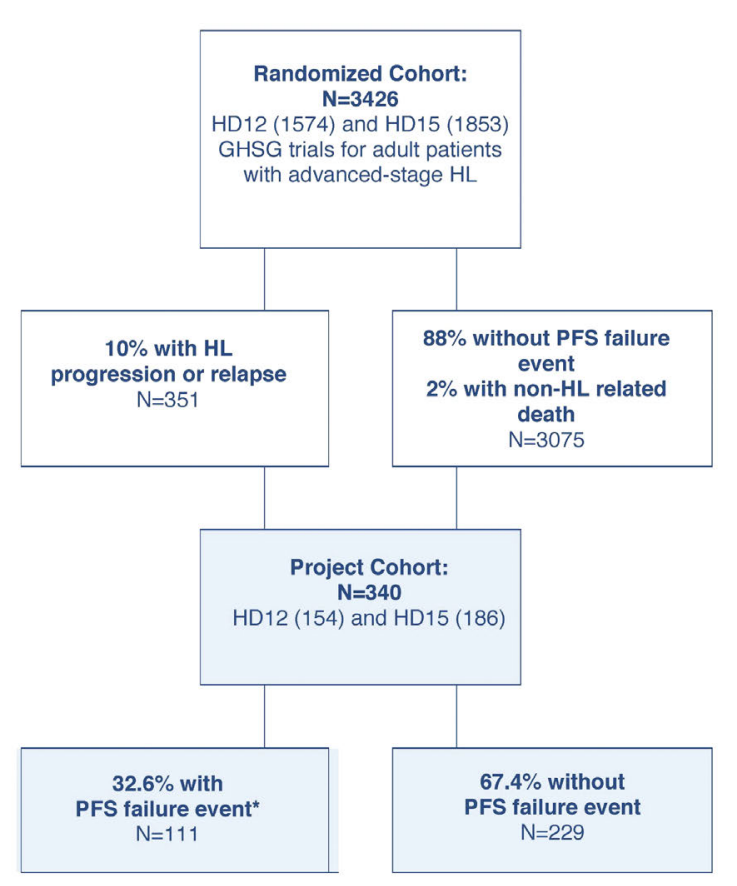

*All events are HL-related

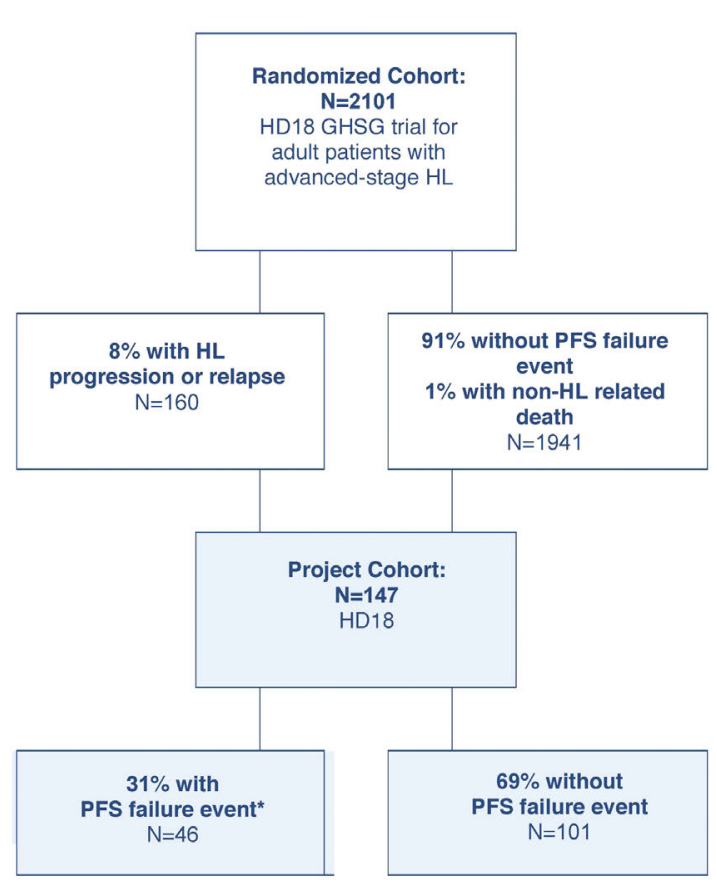

*All events are HL-related

Figure 1. Flowchart of the study cohort and validation cohort. Average values of percentage of the respective cell types are indicated. For calculation of cell counts of macrophages (CD68) and Hodgkin and Reed-Sternberg cells (CD30) see the Online Supplementary Methods.

deviation [SD]: $80.84 \mathrm{~mm}^{2}$ ) (Online Supplementary Figure S1). Since we included the entire lymph node in the analysis, any heterogeneity of cell distribution did not influence our data. Cutting artifacts, and overstained or unstained areas were manually excluded from the analysis. Adjusting the threshold based on several representative locations on the sample also ensured that the setting for analysis for each sample had been selected to cover the specific staining of the lymph node. See the Online Supplementary Methods for a detailed description and statistics.

\section{Results}

\section{Mapping the microenvironment in classical Hodgkin lymphoma through whole-slide image analysis in the study cohort}

Within the study cohort, WSI detected a mean total cell count of 1,550,980 (SD: 949,004) for all analyzed lymph node specimens. The main cellular components of the tumor tissue $\left(\mathrm{CD}^{+} \mathrm{T}\right.$ cells, $\mathrm{CD} 20^{+} \mathrm{B}$ cells, $\mathrm{CD} 30^{+}$HRSC and $\mathrm{CD}^{2} 8^{+}$macrophages) reflect the variable cell proportions dependent on the histological subtype (Figure 2). Overall, T cells, B cells, HRSC and macrophages accounted for a mean of $80 \%$ of all cells within the tumor tissue, highlighting the strength of WSI for comprehensively assessing cHL tumors. As expected, lymphocyte-rich cHL $(n=9)$ showed high counts of B cells and T cells with concomitant low levels of HRSC and macrophages. Conversely, lymphocyte-depleted $\mathrm{cHL}(\mathrm{n}=4)$ displayed the lowest counts of B cells and T cells while CD30 and CD68 levels were markedly elevated. The T-cell content displayed a moderate positive correlation with B-cell content $(\rho=0.42 ; P<0.001)$, as well as a weak correlation with macrophage content $(\rho=0.15 ; P=0.0049)$ and CD30 content $(\rho=0.15 ; P=0.0063)$ (Table 2$)$. We also observed a weak relationship between macrophage and HRSC content $(\rho=0.21 ; P=0.0001)$. Of note, we did not detect a correlation between $\mathrm{B}$-cell and macrophage content.

\section{B-cell content was associated with risk of progression and relapse in the study cohort}

We analyzed whether CD3, CD20, CD30 and CD68 differed between patients with lasting complete remission and patients with subsequent progression or relapse. While we did not observe any association of T-cell count, macrophage content or CD30-positivity with long-term remission status in our study cohort, B-cell content was significantly lower at the time of diagnosis in patients with later progression or relapse (mean 13.5\%) than in patients with long-lasting complete remission (mean: $13.5 \%$ vs. $17.7 \%$, respectively; $P=0.0079$ ) (Table 3 ). Further subgroup analyses revealed that patients with mixed cellularity and nodular sclerosis cHL had significantly lower B-cell counts when at risk for relapse and a similar trend was observed for patients with lymphocytedepleted and lymphocyte-rich cHL (data not shown).

To further assess the association between B-cell content and progression-free survival, we performed explorative receiver operating characteristic (ROC) analyses in which a B-cell content of $21 \%$ or less was the best predictor of progression or relapse in the study cohort (ROC estimate - chance $=0.00959, P<0.0001)$. Utilizing this cut-off, both progression-free survival $(P=0.0004$, hazard ratio $[\mathrm{HR}]=2.479,95 \% \mathrm{CI}: 1.479-4.157)$ and overall survival 
$(P=0.04, \mathrm{HR}=2.372,95 \% \mathrm{CI}:$ 0.996-5.651) were significantly worse in patients presenting with low B-cell content (Figures $3 \mathrm{~A}$ and $4 \mathrm{~A}$ ). We next examined the prognostic value of $B$-cell content in a multivariate model, including 11 known risk factors for progression-free survival and B-cell content as binary variables. Backward selection of effects with $P$-values $<0.1$ resulted in elimination of nine effects (extranodal disease, lymphopenia, stage IV disease, albumin $<4 \mathrm{~g} / \mathrm{dL}$, leukocytosis, B-symptoms, elevated erythrocyte sedimentation rate, hemoglobin $<10.5 \mathrm{~g} / \mathrm{dL}$, large mediastinal mass) and a final model including $\mathrm{B}$-cell content $\leq 21 \% \quad(P=0.0002$, odds ratio $[\mathrm{OR}]=3.133,95 \% \mathrm{CI}$ : 1.726-5.687), male sex $(P=0.0015, \mathrm{OR}=2.251,95 \% \mathrm{CI}$ : $1.363-3.720)$ and age $\geq 45$ years $(P=0.056, O R=1.837,95 \%$ CI: $0.985-3.425)$ as risk factors for treatment failure.

\section{B-cell content was associated with progression-free and overall survival in an Independent validation cohort}

To validate whether low B-cell content at diagnosis, as determined by WSI, could predict survival in an independent validation cohort, we analyzed 147 patients treated within the HD18 trial. ${ }^{21}$ In line with the results observed in our study cohort, B-cell content at diagnosis was lower in patients who had progressive disease than in patients with long-lasting complete remission (mean $16.4 \%$ vs. $21.5 \%$, respectively; $P=0.0616$ ) (Table 3 ). cHL patients in the validation cohort with a low B-cell content (most stringent cut-off value of $10 \%$ ) had a worse progression-free survival ( $P=0.04, \mathrm{HR}=1.981,95 \% \mathrm{CI}: 1.104-3.557)$ (Figure $3 \mathrm{~B})$ and inferior overall survival $(P=0.01, \mathrm{HR}=3.598,95 \%$ CI: 1.205-10.739) compared to patients with a high B-cell count (Figure 4B).

\section{Phenotype and distribution of B cell in classical Hodgkin lymphoma}

$B$ cells and macrophages were extremely unevenly distributed over lymph node tissues but did not reflect unaf-

Table 1A. Patients' baseline characteristics comparing the study cohort and all patients randomized into HD12 and HD15 GHSG trials.

\begin{tabular}{|c|c|c|c|}
\hline Patients' characteristics & $\begin{array}{l}\text { Study cohort } \\
(n=340)\end{array}$ & $\begin{array}{c}\text { Randomized } \\
\text { cohort } N=3426\end{array}$ & P* \\
\hline Age in years - median (IQR) & $31(24-40)$ & $33(25-42)$ & $0.0093^{* *}$ \\
\hline $\begin{array}{l}\text { GHSG risk factors, } n(\%) \\
\text { Extranodal disease } \\
\geq 3 \text { nodal areas } \\
\text { Large mediastinal mass } \\
\text { Elevated ESR }\end{array}$ & $\begin{array}{l}64(18.8) \\
304(89.4) \\
106(31.2) \\
248(72.9)\end{array}$ & $\begin{array}{c}693(20.3) \\
2884(84.3) \\
1024(30.1) \\
2310(69.5)\end{array}$ & $\begin{array}{l}0.5226 \\
0.0058 \\
0.6631 \\
0.1532\end{array}$ \\
\hline $\begin{array}{l}\text { IPS risk factors, } \mathrm{n}(\%) \\
\text { Male sex } \\
\text { Age } \geq 45 \text { years } \\
\text { Clinical stage IV } \\
\text { Albumin }<4 \mathrm{~g} / \mathrm{dL} \\
\text { Hemoglobin }<10.5 \mathrm{~g} / \mathrm{dL} \\
\text { Leukocytosis } \\
\text { Lymphopenia }\end{array}$ & $\begin{array}{c}205(60.3) \\
53(15.6) \\
142(41.8) \\
192(56.5) \\
56(16.5) \\
77(22.6) \\
23(6.8)\end{array}$ & $\begin{array}{c}2059(60.1) \\
719(21.0) \\
1198(35.0) \\
1796(55.1) \\
617(18.1) \\
700(20.5) \\
261(8.0)\end{array}$ & $\begin{array}{l}0.9535 \\
0.0094 \\
0.0069 \\
0.6044 \\
0.4579 \\
0.3220 \\
0.4586\end{array}$ \\
\hline $\begin{array}{l}\text { IPS score, n(\%) } \\
0-2 \\
3-7\end{array}$ & $\begin{array}{l}204(60.0) \\
136(40.0)\end{array}$ & $\begin{array}{l}1721(55.8) \\
1069(34.6)\end{array}$ & 0.5553 \\
\hline $\begin{array}{l}\text { Histological subtype, n(\%) } \\
\text { Nodular sclerosis } \\
\text { Mixed cellularity } \\
\text { Lymphocyte-rich } \\
\text { Lymphcyte-depleted } \\
\end{array}$ & $\begin{array}{c}221(65.0) \\
100(29.4) \\
9(2.6) \\
4(1.2)\end{array}$ & $\begin{array}{c}1778(51.9) \\
780(22.8) \\
73(2.1) \\
35(1.0) \\
\end{array}$ & $\begin{array}{c}<0.0001 \\
0.0027 \\
0.4319 \\
0.7734 \\
\end{array}$ \\
\hline
\end{tabular}

fected tissue (Online Supplementary Figures S2 and S3). Visual inspection by experienced pathologists revealed non-infiltrated, pre-existing tissue in only $15 \%$ of cases $(24 / 154)$ and the non-infiltrated tissues in these cases usually represented only a small fraction of the lymph node (median unaffected area in not-completely infiltrated cases, 10\%). Furthermore, we did not detect an association of B-cell content by WSI and stage of disease for stages included in this cohort (stages II, III and IV; data not shown). Since WSI subsumes B cells in the whole lymph node, including B-cell nodules with considerable distance to HRSC (Online Supplementary Figure S4), we tested an association with B-cell content in the immediate vicinity of HRSC ( $<100 \mu \mathrm{m}$ radius) (see Online Supplementary Methods). Interestingly, despite the uneven distribution of $B$ cells in the lymph node, we observed a correlation between B-cell content in the whole lymph node and immediate proximity to HRSC $(\rho=0.676, P<0.0001, n=41)$ (Online Supplementary Figure S5). B cells at the borders of the nodular infiltration by HRSC are arranged in illdefined follicles that lack germinal centers and are composed mainly of IgD-positive follicle mantle cells, some of

Table 1B. Patients' baseline characteristics comparing the validation cohort and all patients randomized into the HD18 GHSG trial.

\begin{tabular}{|c|c|c|c|}
\hline $\begin{array}{l}\text { Patients' characteristics } \\
\text { of validiation cohort }\end{array}$ & $\begin{array}{c}\text { Validation } \\
\text { cohort }(N=147)\end{array}$ & $\begin{array}{c}\text { Randomized } \\
\text { cohort (N=2101) }\end{array}$ & $P$ \\
\hline Age in years, median (IQR) & $33(25-42)$ & $32(24-43)$ & 0.8322 \\
\hline $\begin{array}{l}\text { GHSG risk factors, } \mathrm{n}(\%) \\
\text { Extranodal disease } \\
\geq 3 \text { nodal areas } \\
\text { Large mediastinal mass } \\
\text { Eelevated ESR }\end{array}$ & $\begin{array}{c}28(19.0) \\
128(87.1) \\
37(25.2) \\
93(63.3)\end{array}$ & $\begin{array}{c}407(19.4) \\
1809(86.1) \\
592(28.2) \\
1332(63.4)\end{array}$ & $\begin{array}{c}1 \\
0.8053 \\
0.4473 \\
0.9295\end{array}$ \\
\hline $\begin{array}{l}\text { IPS risk factors, } \mathrm{n}(\%) \\
\text { Male sex } \\
\text { Age } \geq 45 \text { years } \\
\text { Clinical stage IV } \\
\text { Albumin }<4 \mathrm{~g} / \mathrm{dL} \\
\text { Hemoglobin }<10.5 \mathrm{~g} / \mathrm{dL} \\
\text { Leukocytosis } \\
\text { Lymphopenia }\end{array}$ & $\begin{array}{c}96(66.0) \\
26(17.7) \\
53(36.1) \\
85(87.5) \\
28(19.0) \\
30(20.4) \\
10(6.8)\end{array}$ & $\begin{array}{c}1278(60.8) \\
437(22.4) \\
765(36.4) \\
1180(56.2) \\
379(18.0) \\
436(20.8) \\
151(7.2)\end{array}$ & $\begin{array}{c}0.1900 \\
0.2156 \\
1 \\
0.7305 \\
0.7390 \\
1 \\
1\end{array}$ \\
\hline $\begin{array}{l}\text { IPS score, n(\%) } \\
\quad 0-2 \\
3-7\end{array}$ & $\begin{array}{l}92(63.0) \\
54(37.0)\end{array}$ & $\begin{array}{c}1206(61.9) \\
741(38.1)\end{array}$ & 0.8598 \\
\hline $\begin{array}{l}\text { Histological subtype - N/125 } \\
\text { Nodular sclerosis } \\
\text { Mixed-cellularity } \\
\text { Lymphocyte-rich } \\
\text { Lymphcyte-depleted }\end{array}$ & $\begin{array}{c}72(49.0) \\
25(17.0) \\
4(2.7) \\
0 \\
\end{array}$ & $\begin{array}{c}747(35.6) \\
245(11.7) \\
29(1.4) \\
8(0.4)\end{array}$ & $\begin{array}{c}0.0006 \\
0.0447 \\
0.1402 \\
1\end{array}$ \\
\hline
\end{tabular}

HD12 trial: number of patients per arm (study cohort/randomized cohort): arm A 39/336, arm B 59/357, arm C 47/353, arm D 44/349. Chemotherapy: arms A and B: 8 cycles of escalated BEACOPP (eBEACOPP), arms C and D: 4 cycles of eBEACOPP + 4 cycles of baseline BEACOPP. Radiotherapy: arms A and D: 30 Gy to initial bulky sites and residual lymphoma. HD15 trial: number of patients per arm: arm A 64/677, arm B 69/680, arm C 73/674. Chemotherapy: arm A 8 cycles of eBEACOPP, arm B: 6 cycles of eBEACOPP, arm C: 8 cycles of BEACOPP14 Radiotherapy: arms A, B, and C: 30 Gy consolidating radiotherapy to positron emission tomography (PET)-positive residues. HD18 trial: number of patients per arm (validation cohort/enrolled or randomized cohort): NULL 9/137, arm A 67/731 arm B 15/220, arm C 27/508, arm D 29/505. NULL not randomized arm: without PET result after 2 cycles of eBEACOPP Chemotherapy for PET-positive arms A: 6-8 cycles of eBEACOPP and B: 6-8 cycles of eBEACOPP + rituximab; and for PET-negative arms C: 6-8 cycles of eBEACOPP and D: 4 cycles of eBEACOPP. Radiotherapy (30 Gy) was recommended for all arms with residual ${ }^{18} \mathrm{~F}-\mathrm{FDG}$ uptake and a mass with a largest diameter after chemotherapy of at least $2.5 \mathrm{~cm} . P$ values are for the comparison of patients included in the study or validation cohort versus patients not included. *Fisher exact test, if not stated otherwise, **Wilcoxon rank-sum test, IQR: interquartile range; ESR: erythrocyte sedimentation rate; IPS: International Prognostic Score: GHSG: German Hodgkin Study Group. 


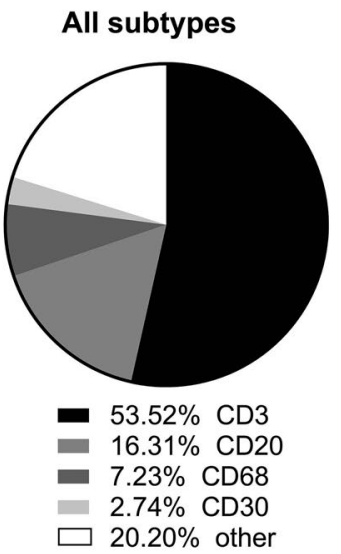

lymphocyte-rich

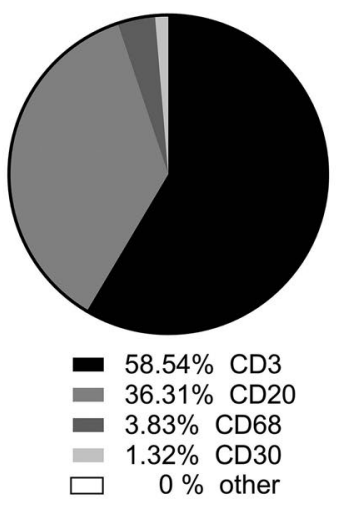

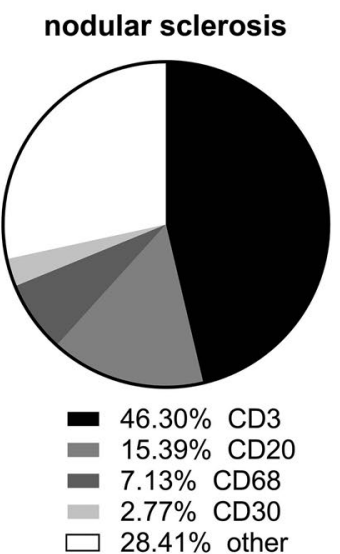

lymphocyte-depleted

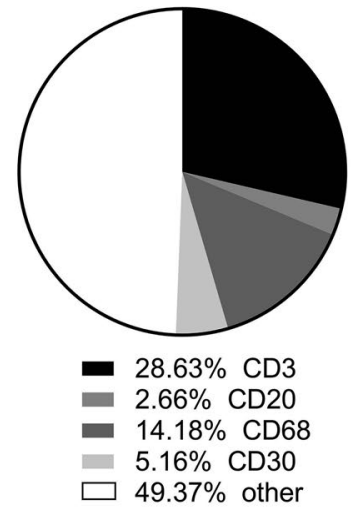

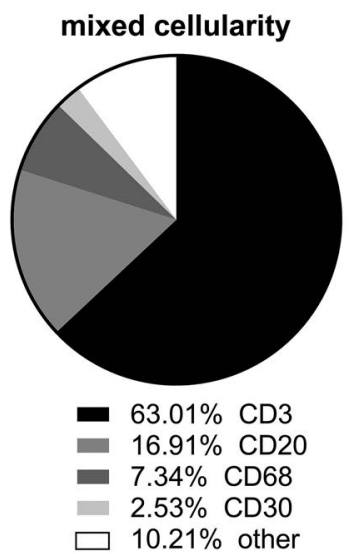

not specified

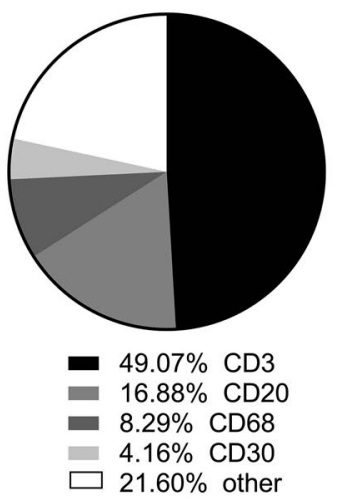

Figure 2. Cellular composition assessed by whole-slide imaging in the study cohort and according to histological subtypes of classical Hodgkin lymphoma. Average values of percentage of the respective cell types are indicated. For calculation of cell counts of macrophages (CD68) and Hodgkin and Reed-Sternberg cells (CD30) see the Online Supplementary Methods. which express CD73 (Online Supplementary Figure S4). Thus, the vast majority of $B$ cells seem to resemble naive $\mathrm{B}$ cells. However, further subtyping of $\mathrm{B}$ cells in the microenvironment using appropriate methods is required to understand the nature of this population.

\section{Inter-observer bias of whole-slide image analysis}

To evaluate the inter-dependent bias of WSI, we took a two-pronged approach. First, we randomly selected CD20stained whole slides that were re-analyzed by a second independent observer, using the same software. We found a high inter-observer concordance when the same or newly scanned images were processed in the image analysis software by a second observer (Online Supplementary Figure S6). Second, we randomly selected a cohort of 20 cases, for which the complete staining procedure for CD20 and WSI was performed in a second center (see Online Supplementary Methods). This approach revealed a correlation of results obtained at two independent centers $\left(r^{2}=0.6101\right)$ (Online Supplementary Figure S7). Thus, WSI provides an unbiased approach for robust quantification of the global cellular composition of cHL tissue.

\section{Discussion}

Despite the overall outstanding treatment results in $\mathrm{cHL}$, the a priori identification of a high-risk subset of patients remains a challenge in clinical practice. Studies utilizing functional imaging influence clinical decisionmaking, ${ }^{22}$ but none of the previously proposed gene expression profiling or immunohistochemistry biomarkers has been incorporated into treatment protocols for cHL. ${ }^{4}$ So far, a comprehensive analysis of the cellular composition of the microenvironment in cHL is limited to gene expression studies. . $^{12,1,23}$ Here, we utilized WSI and achieved a comprehensive and robust quantification of the cellular composition of the cHL microenvironment throughout the whole tumor sample. To the best of our knowledge, this approach has not been applied to cHL previously and opens a novel conceptual window into the assessment of cellular composition of tumor tissue.

In contrast to previously reported studies, ${ }^{11,17,24}$ but in line with several other studies, ${ }^{25,26}$ we did not observe a correlation of macrophage content with outcome in cHL. The discrepancy between our results and previously published studies with respect to macrophage counts may be explained in multiple ways. First, the technology to assess macrophage counts differs. We cannot rule out that gene expression analysis utilizing mRNA expression level of multiple genes assesses macrophages in a different manner than our WSI approach. However, comparing conventional immunohistochemistry image analysis or even visual inspection of small fields of view, we consider WSI a more accurate measure of macrophage content. Second, there is a difference between the population of patients in our study and those in previous publications. Patients in our study were treated with rather intensive chemotherapy (BEACOPP/eBEACOPP), which is known to achieve 
Table 2. Correlation analyses of whole-slide image analysis-determined CD3, CD20, CD30 and CD68 content in the study cohort.

\begin{tabular}{|c|c|c|c|c|}
\hline & $\begin{array}{c}\text { CD68-positive area rate } \\
\text { Rho / } P\end{array}$ & $\begin{array}{c}\text { CD20-positive cell rate } \\
\text { Rho / P }\end{array}$ & $\begin{array}{c}\text { CD3-positive cell rate } \\
\text { Rho / } P\end{array}$ & $\begin{array}{c}\text { CD30-positive area rate } \\
\text { Rho / } \mathbf{P}\end{array}$ \\
\hline $\begin{array}{l}\text { CD68-positive area } \\
\text { rate }\end{array}$ & 1 & $\begin{array}{l}-0.05289 \\
0.3389\end{array}$ & $\begin{array}{l}0.15472 \\
0.0049\end{array}$ & $\begin{array}{l}0.21218 \\
0.0001\end{array}$ \\
\hline $\begin{array}{l}\text { CD20-positive cell } \\
\text { rate }\end{array}$ & $\begin{array}{l}-0.05289 \\
0.3389\end{array}$ & 1 & $0.42374 /<0.0001$ & $-0.07154 / 0.1956$ \\
\hline $\begin{array}{l}\text { CD3-positive cell } \\
\text { rate }\end{array}$ & $\begin{array}{l}0.15472 \\
0.0049\end{array}$ & $0.42374 /<0.0001$ & 1 & $0.15024 / 0.0063$ \\
\hline $\begin{array}{l}\text { CD30-positive area } \\
\text { rate }\end{array}$ & $\begin{array}{l}0.21218 \\
0.0001\end{array}$ & $-0.07154 / 0.1956$ & $0.15024 / 0.0063$ & 1 \\
\hline
\end{tabular}

Rho: Pearson correlation coefficients; $P=$ probability $>|r|$ under the null hypothesis: $R h o=0$.

Table 3. Whole-slide image analysis-determined CD20-positive cell rates at baseline according to progression-free survival-failure status.

\begin{tabular}{|c|c|c|c|c|c|c|c|c|}
\hline & \multicolumn{8}{|c|}{ CD20-positive cell rates (\%) } \\
\hline & \multicolumn{4}{|c|}{ Study cohort } & \multicolumn{4}{|c|}{ Validation cohort } \\
\hline & PFS fallure & $N(\%)$ & Group mean & P-value & PFS fallure & $N(\%)$ & Group mean & P-value \\
\hline \multirow[t]{2}{*}{ All patients } & no & $229(67.4)$ & 17.7 & \multirow[t]{2}{*}{0.0079} & no & $201(69.4)$ & 21.5 & \multirow[t]{2}{*}{0.0616} \\
\hline & yes & $111(32.6)$ & 13.5 & & yes & $45(30.6)$ & 16.4 & \\
\hline \multirow[t]{2}{*}{ CD20-negative } & no & $184(67.4)$ & 18.1 & \multirow[t]{2}{*}{0.0002} & no & \multirow[t]{2}{*}{ not available } & & \\
\hline & yes & $89(32.6)$ & 12.4 & & yes & & & \\
\hline \multirow[t]{2}{*}{ CD20-positive } & no & $45(67.2)$ & 16.3 & \multirow[t]{2}{*}{ n.s. } & no & \multirow[t]{2}{*}{ not available } & & \\
\hline & yes & $22(32.8)$ & 17.9 & & yes & & & \\
\hline \multirow[t]{2}{*}{ Mixed cellularity } & no & $70(70.0)$ & 19.5 & \multirow[t]{2}{*}{0.0064} & no & $20(80)$ & 20.8 & \multirow[t]{2}{*}{0.2529} \\
\hline & yes & $30(30.0)$ & 13.3 & & yes & $5(20)$ & 14.4 & \\
\hline \multirow[t]{2}{*}{ Nodular sclerosis } & no & $145(65.6)$ & 16.9 & \multirow[t]{2}{*}{0.0033} & no & $145(65.6)$ & 16.9 & \multirow[t]{2}{*}{0.0274} \\
\hline & yes & $76(34.4)$ & 12.1 & & yes & $76(34.4)$ & 12.1 & \\
\hline \multirow[t]{2}{*}{ Lymphocyte-depleted } & no & $3(0.75)$ & 2.4 & \multirow[t]{2}{*}{ n.s. } & no & 0 & 0 & \multirow{2}{*}{$\begin{array}{c}\text { not } \\
\text { applicable }\end{array}$} \\
\hline & yes & $1(0.25)$ & 5.5 & & yes & 0 & 0 & \\
\hline \multirow[t]{2}{*}{ Lymphocyte rich } & no & $6(0.67)$ & 32.2 & \multirow[t]{2}{*}{ n.s. } & no & $4(100)$ & 18.2 & \multirow{2}{*}{$\begin{array}{c}\text { not } \\
\text { applicable }\end{array}$} \\
\hline & yes & $3(0.33)$ & 55.7 & & yes & 0 & & \\
\hline
\end{tabular}

PFS: progression-free survival.

higher levels of event-free survival than the levels achieved with ABVD-based regimens. ${ }^{21}$ However, the prognostic relevance of macrophage content was absent despite the fact that we enriched the cohort for patients who had events signifying progression. It is important to mention that we excluded all non-cHL and all non-treatment-related deaths ( $n=55$ in the study cohort) from all the analyses in order to identify a biomarker of lymphoma aggressiveness that was not biased by unrelated deaths. However, inclusion of those 55 patients as progressionfree survival failures did not affect the overall results obtained within this study (data not shown).

One might speculate that the BEACOPP regimen reduced lymphoma-related events and that biomarkers established in patients treated with ABVD might lose their prognostic power. Recently published results on the prognostic range of the IPS in patients with advanced-stage cHL enrolled on the Eastern Cooperative Oncology Group 2496 trial do in fact argue for such an interpretation. ${ }^{27}$ In the aforementioned study, two factors (age and stage) were significantly associated with freedom from progression in a multivariate analysis. ${ }^{27}$ Similarly, we found that apart from low B-cell content, only male sex and age were predictive of treatment failure in a multivariate analysis. Similar findings were reported by the Spanish Hodgkin Lymphoma Study Group in an analysis of patients with advanced-stage $\mathrm{cHL}$ treated with $\mathrm{ABVD}{ }^{28}$ We thus believe that our study cohort is representative of the population of advanced-stage cHL patients and the absence of a prognostic significance of macrophage content is most likely due to the effective treatment applied.

In light of the fact that the BEACOPP-treated populations analyzed in our study had very few lymphoma-related events (progression or relapse), the value of CD20 content by WSI as a prognostic tool appears to be even higher. $\mathrm{B}$-cell content in the microenvironment of $\mathrm{cHL}$ has been identified in several previous studies as a prognostic tool if analyzed by gene expression profiling or immunohistochemistry. ${ }_{12,16,17,29,30}$ However, this biomarker has so far attracted less attention compared to the macrophage count. Gene expression profiling of the $\mathrm{cHL}$ microenvironment is a laborious technology that is not widely available. In addition, gene expression analysis is dependent on the technology applied, leading to the different prognostic expression patterns in multiple studies. ${ }^{12,16,31}$ Unfortunately, immunohistochemistry techniques, which are theoretically easy to apply in a routine diagnostic setting, are mostly used to analyze small areas or even hotspots of B cells, making this approach prone to observer-dependent bias. ${ }^{17,30}$ Nevertheless, several groups previously noted that the expression of B-cell cluster genes was related to a favorable outcome in cHL. ${ }^{12,16,17,29,30}$ In contrast to previous findings, we did not identify a correlation between B-cell and macrophage content, which might also be related to the methodology used.

In view of the recent demonstration of variable pheno- 
A Project cohort

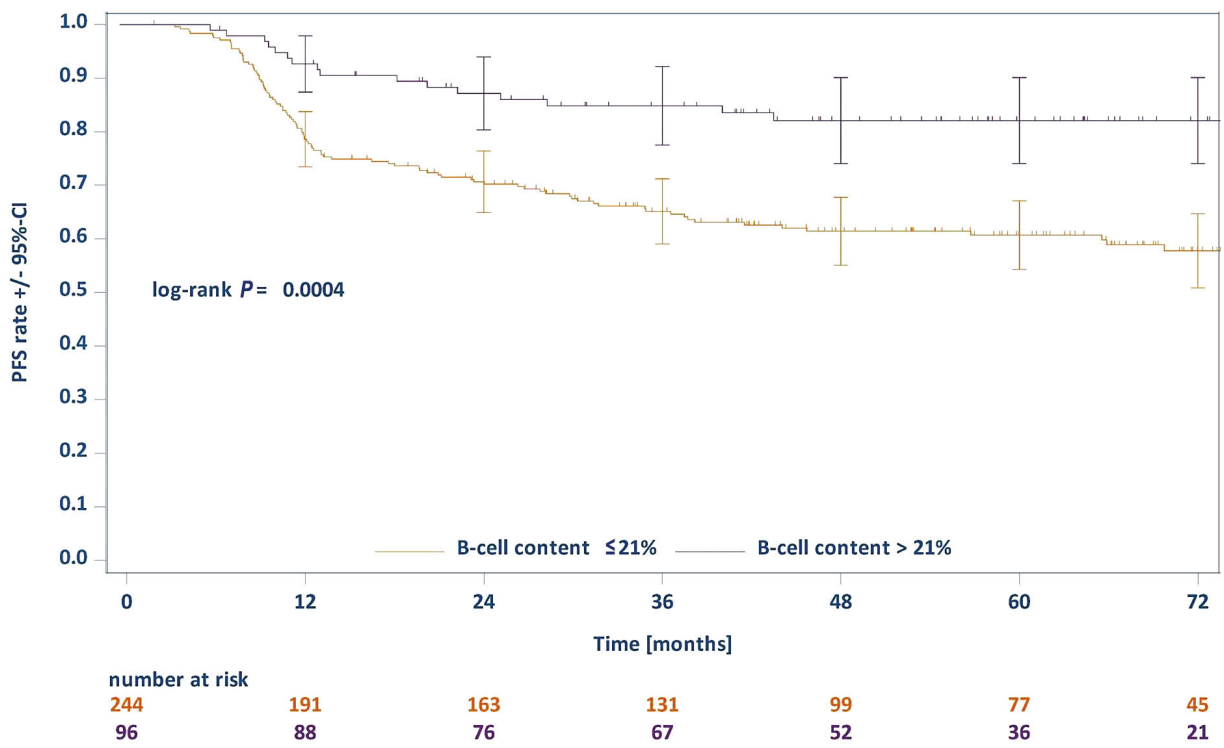

Figure 3. Progression-free survival according to CD20 content. (A) Kaplan-Meier plots of progression-free survival (PFS) in the study cohort for the two risk groups according to B-cell content (CD20-positive cell rate: $\leq 21 \%$ or $>21 \%)$. (B) Kaplan-Meier plots of PFS in the validation cohort for the two risk groups according to $\mathrm{B}$ cell content (CD20-positive cell rate: $\leq 10 \%$ or $>10 \%$ ).

B Validation cohort

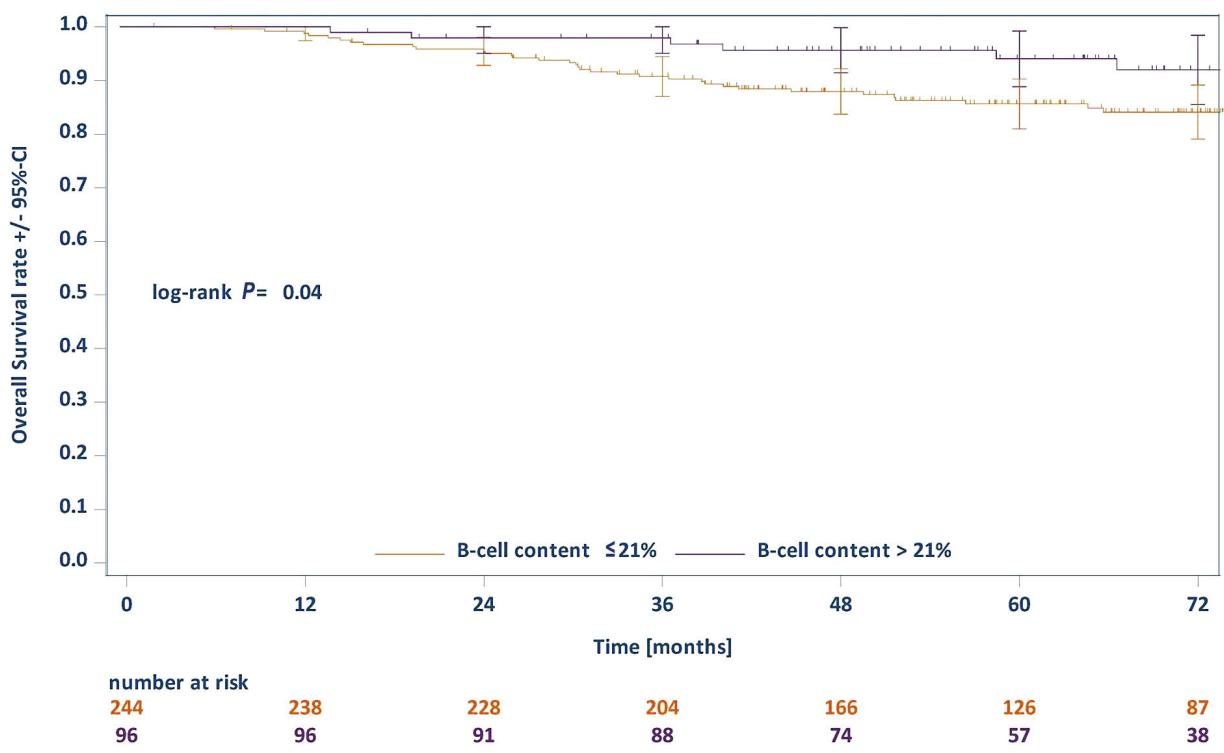

types of tumor-infiltrating B cells populating the microenvironment of solid cancers, ${ }^{32}$ the adoption of a broader panel of B-cell/plasma-cell-associated antigens, T-cell subsets and correlation with PD-1 staining should be performed in future WSI applications and could include, among others, CD4, CD8, CD19, CD27, CD5, CD38 and CD138. However, WSI on large cohorts, as performed in our study, will probably have to be focused on a limited number of markers.

Nevertheless, WSI has several advantageous features, compared to gene expression profiling and conventional immunohistochemistry studies, by combining the diagnostic accuracy of digital image analysis and a large-scale approach. By providing cell counts (e.g., the number of B cells) independently of relative expression levels of biomarkers for cell subtypes (e.g., CD20 mRNA), WSI reflects a direct read-out for the cellular composition and consequently a direct measure for therapeutic targets of immunological therapy. Since CD20 staining is performed at virtually any diagnosis of cHL, WSI makes use of existing histology data without any additional molecular procedures. CD20-stained slides of cHL can be digitalized at any pathology center and moved via the worldwide web in a timely manner to allow centralized assessment. We thus envision this technology to be highly suitable for incorporation into future clinical trials.

The cellular composition of the microenvironment of cHL is complex and its analysis has so far been restricted to a limited number of lymphoma specimens. ${ }^{33}$ WSI of multiplexed-stains allows the number of cellular markers to be increased and might help in the translation of findings obtained in a few patients' specimens into large cohorts of patients. Moreover, novel analytic tools beyond the plain assessment of relative amount of cell types can potentially be applied to WSI data. ${ }^{34}$ In summary, B-cell content assessed by WSI in advanced-stage cHL allows for 
A Project cohort

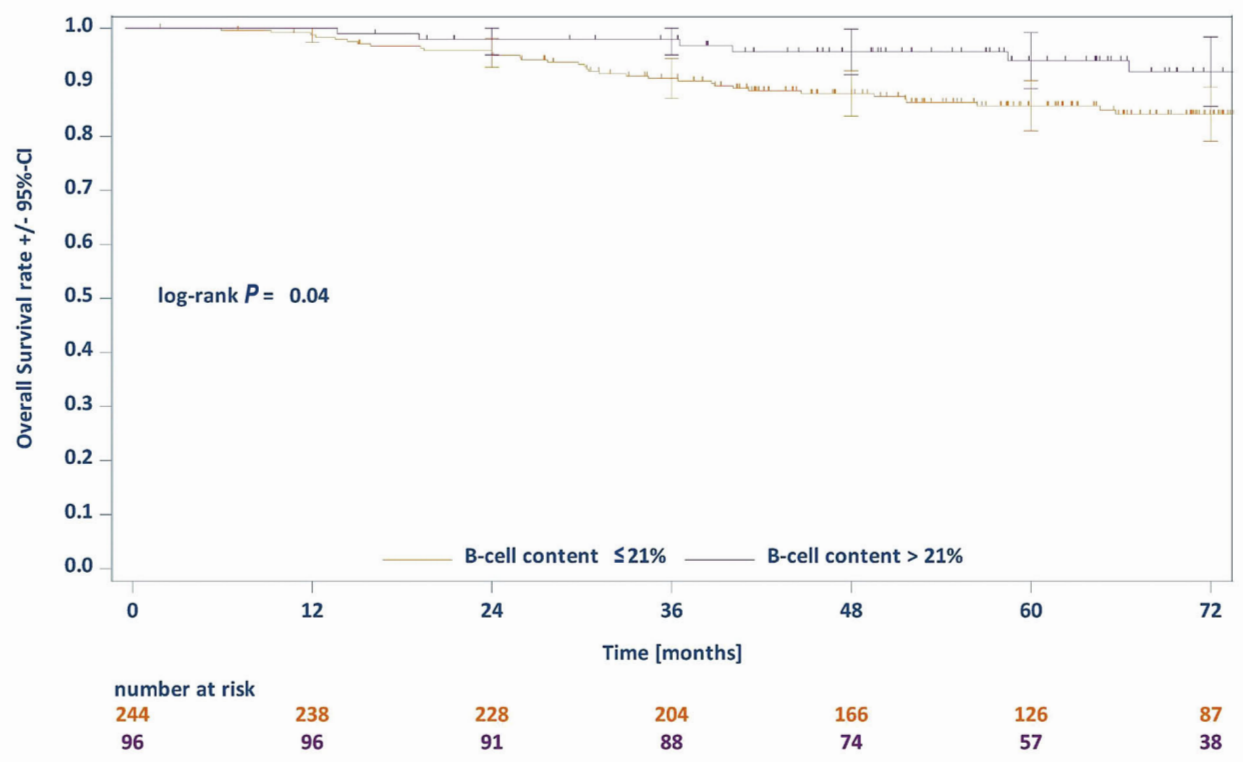

Figure 4. Overall survival according to CD20 content. (A) Kaplan-Meier plots of overall survival in the study cohort for the two risk groups according to B-cell content (CD20positive cell rate: $\leq 21 \%$ or $>21 \%$ ). (B) Kaplan-Meier plots of overall survival in the validation cohort for the two risk groups according to B-cell content (CD20-positive cell rate: $\leq 10 \%$ or $>10 \%$ ).

B Validation cohort

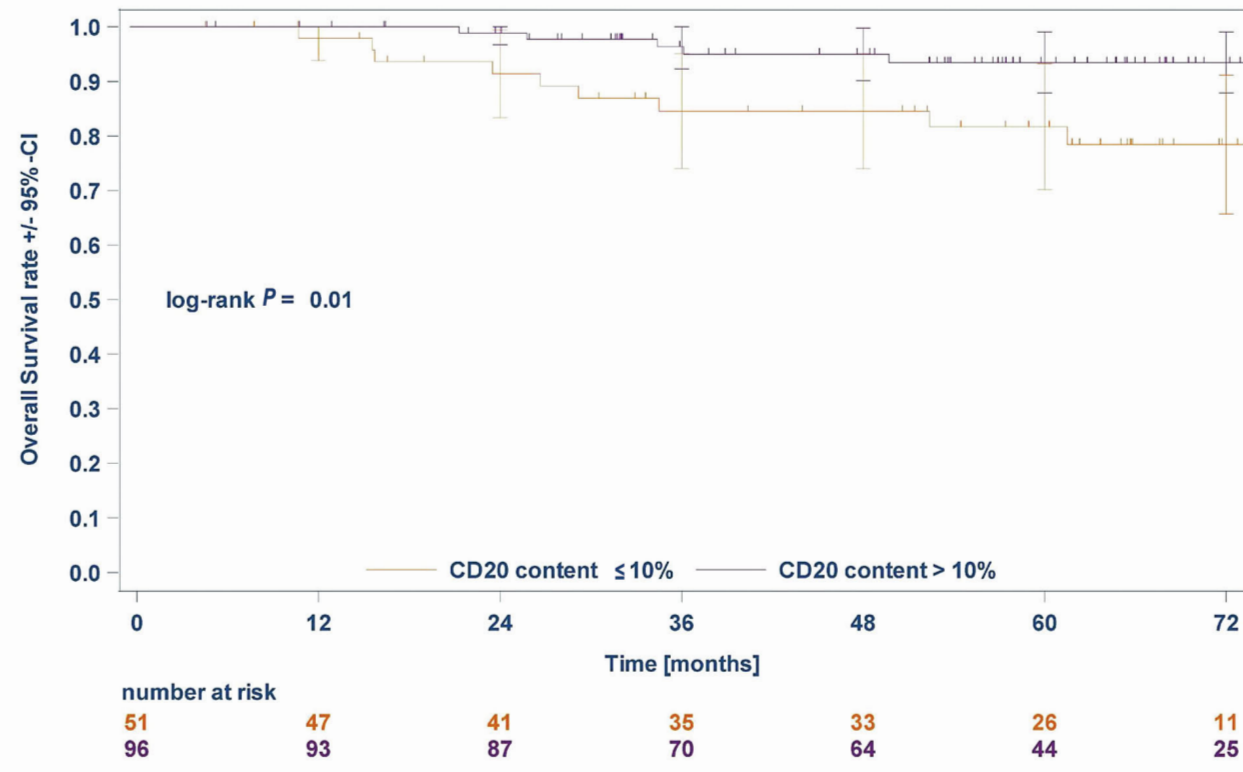

a robust discrimination of patients at high risk of experiencing relapse or progressive disease and thus identifies a population of patients who may qualify for novel first-line treatment strategies. Furthermore, we envision that WSI may also be applied to identify patients in whom de-escalation of treatment intensity may be possible. We thus anticipate the use of WSI in all future GHSG studies. Even though additional testing is required to define cut-off values, this approach is close to clinical application since the data required (CD20 staining) are generated in the standard diagnostic workup of any cHL around the world. We cannot imagine any other technology with such a broad potential for application, considering that even in less well-developed countries access may be affordable.
Finally, this is a unique opportunity to establish a risk model looking specifically at the microenvironment in prospective clinical trials.

\section{Disclosures}

$A E$ : consultancy or advisory services for and honoraria from Takeda and BMS; research funding from Takeda, BMS and Affimed; honoraria. HCR: consultancy or advisory services for Abbvie and AstraZeneca; research funding from Gilead Sciences. BvT: consultancy or advisory services for Amgen, Pfizer, Takeda and MSD; honoraria from Roche, Takeda and MSD; research funding from Novartis, MSD and Takeda. WK: consultancy or advisory services for and honoraria from Takeda; research funding from Takeda, Amgen, Regeneron and Roche. 


\section{Contributions}

$R D J, A P, H H, S R, A E$ and $W K$ designed the research. $R D J, L P$ SR, AG, AP, HH, LF, FF, MO, FJ, CT, MLH, PM, AR, HS $H C R, P B, B v T, A E$ and $W K$ collected and assembled the data. $R D J, S R, A P, A E$ and WK analyzed and interpreted the data. RDJ and WK wrote the first draft and all authors approved the paper.

\section{Acknowledgments}

The authors thank all patients enrolled in GHSG clinical trials, all clinicians and pathologists supporting the clinical trials and the translational research program of the GHSG.

\section{Funding}

This work was supported by a grant from German Cancer Aid (Deutsche Krebshilfe n. 70112502), the Else Kröner-Fresenius Stiftung (2016-Kolleg-19 to RDJ) and the Stiftung Kölner Krebsforschung (to RDJ). Image analysis software was supported by the Kinderkrebsinitiative Buchholz, Holm-Seppensen.

\section{References}

1. Diehl V, Franklin J, Pfreundschuh $\mathrm{M}$, et al. Standard and increased-dose BEACOPP chemotherapy compared with COPPABVD for advanced Hodgkin's disease. $\mathrm{N}$ Engl J Med. 2003;348(24):2386-2395.

2. Sjoberg J, Halthur C, Kristinsson SY, et al. Progress in Hodgkin lymphoma: a population-based study on patients diagnosed in Sweden from 1973-2009. Blood. 2012; 119(4):990-996.

3. Goodman KA, Riedel E, Serrano V, et al. Long-term effects of high-dose chemotherapy and radiation for relapsed and refractory Hodgkin's lymphoma. I Clin Oncol. 2008;26(32):5240-5247.

4. Eichenauer DA, Engert A, Andre $M$, et al. Hodgkin's lymphoma: ESMO Clinical Practice Guidelines for diagnosis, treatment and follow-up. Ann Oncol. 2014;25(Suppl 3):iii70-75.

5. Aoki T, Steidl C. Novel biomarker approaches in classic Hodgkin lymphoma. Cancer J. 2018;24(5):206-214.

6. Condeelis J, Pollard JW. Macrophages: obligate partners for tumor cell migration, invasion, and metastasis. Cell. 2006;124(2):263266

7. Vassallo J, Metze K, Traina F, et al. Further remarks on the expression of CD20 in classical Hodgkin's lymphomas. 2002;87(3): ELT17.

8. Rassidakis GZ, Medeiros LJ, Viviani S, et al. CD20 expression in Hodgkin and ReedSternberg cells of classical Hodgkin's disease: associations with presenting features and clinical outcome. J Clin Oncol. 2002;20(5):1278-1287.

9. Aldred V, Vassallo J, Froes MCAH, Augusto Soares F. CD20 expression by HodgkinReed-Sternberg cells in classical Hodgkin lymphoma is related to reduced overall survival in young adult patients. Leuk Lymphoma. 2008;49(11):2198-2202.

10. Portlock CS, Donnelly GB, Oin J, et al. Adverse prognostic significance of CD20 positive Reed-Sternberg cells in classical Hodgkin's disease. $\mathrm{Br} \mathrm{J}$ Haematol. 2004;125(6):701-708.

11. Steidl C, Farinha P, Gascoyne RD. Macrophages predict treatment outcome in Hodgkin's lymphoma. Haematologica. 2011;96(2):186-189.

12. Steidl C, Lee T, Shah SP, et al. Tumor-associated macrophages and survival in classic Hodgkin's lymphoma. N Engl J Med. 2010;362(10):875-885.

13. Tzankov A, Matter MS, Dirnhofer S. Refined prognostic role of CD68-positive tumor macrophages in the context of the cellula micromilieu of classical Hodgkin lymphoma. Pathobiology. 2010;77(6):301-308

14. Kamper P, Bendix K, Hamilton-Dutoit S, et al. Tumor-infiltrating macrophages correlate with adverse prognosis and Epstein-Barr virus status in classical Hodgkin's lymphoma. Haematologica. 2011;96(2):269-276.

15. Panico L, Ronconi F, Lepore $M$, et al. Prognostic role of tumor-associated macrophages and angiogenesis in classical Hodgkin lymphoma. Leuk Lymphoma. 2013;54(11):2418-2425.

16. Chetaille B, Bertucci F, Finetti $\mathrm{P}$, et al. Molecular profiling of classical Hodgkin lymphoma tissues uncovers variations in the tumor microenvironment and correlations with EBV infection and outcome. Blood. 2009;113(12):2765-3775.

17. Greaves P, Clear A, Coutinho R, et al. Expression of FOXP3, CD68, and CD20 at diagnosis in the microenvironment of classical Hodgkin lymphoma is predictive of outcome. J Clin Oncol. 2013;31(2):256-262.

18. Tudor CS, Distel LV, Eckhardt J, et al. B cells in classical Hodgkin lymphoma are important actors rather than bystanders in the local immune reaction. Hum Pathol. 2013;44(11):2475-2486.

19. Borchmann P, Haverkamp H, Diehl V, et al. Eight cycles of escalated-dose BEACOPP compared with four cycles of escalated-dose BEACOPP followed by four cycles of baseline-dose BEACOPP with or without radiotherapy in patients with advanced-stage hodgkin's lymphoma: final analysis of the HD12 trial of the German Hodgkin Study Group. J Clin Oncol. 2011;29(32):4234-4242.

20. Engert A, Haverkamp $H$, Kobe $C$, et al. Reduced-intensity chemotherapy and PETguided radiotherapy in patients with advanced stage Hodgkin's lymphoma (HD15 trial): a randomised, open-label, phase 3 non-inferiority trial. Lancet. 2012:379(9828):1791-1799

21. Borchmann P, Goergen $\mathrm{H}$, Kobe C, et al PET-guided treatment in patients with advanced-stage Hodgkin's lymphoma (HD18): final results of an open-label, international, randomised phase 3 trial by the German Hodgkin Study Group. Lancet. 2018;390(10114):2790-2802.

22. Hutchings M, Loft A, Hansen M, et al. FDG PET after two cycles of chemotherapy predicts treatment failure and progression-free survival in Hodgkin lymphoma. Blood. 2006;107(1):52-59.

23. Scott DW, Chan FC, Hong F, et al. Gene expression-based model using formalinfixed paraffin-embedded biopsies predicts overall survival in advanced-stage classical
Hodgkin lymphoma. J Clin Oncol. 2013;31 (6):692-700

24. Tan KL, Scott DW, Hong F, et al. Tumor associated macrophages predict inferior outcomes in classic Hodgkin lymphoma: a correlative study from the E2496 Intergroup trial. Blood. 2012;120(16):3280-3287.

25. Azambuja D, Natkunam Y, Biasoli I, et al Lack of association of tumor-associated macrophages with clinical outcome in patients with classical Hodgkin's lymphoma. Ann Oncol. 2012;23(3):736-742.

26. Sanchez-Espiridion B, Martin-Moreno AM Montalban C, et al. Immunohistochemica markers for tumor associated macrophages and survival in advanced classical Hodgkin's lymphoma. Haematologica. 2012;97(7): 1080-1084.

27. Diefenbach CS, Li $\mathrm{H}$, Hong $\mathrm{F}$, et al. Evaluation of the International Prognostic Score (IPS-7) and a Simpler Prognostic Score (IPS-3) for advanced Hodgkin lymphoma in the modern era. Br J Haematol. 2015;171 (4):530-538.

28. Guisado-Vasco P, Arranz-Saez R, Canales M et al. Stage IV and age over 45 years are the only prognostic factors of the International Prognostic Score for the outcome of advanced Hodgkin lymphoma in the Spanish Hodgkin Lymphoma Study Group series. Leuk Lymphoma. 2012;53(5):812-819.

29. Sanchez-Aguilera A, Montalban C, de la Cueva $\mathrm{P}$, et al. Tumor microenvironment and mitotic checkpoint are key factors in the outcome of classic Hodgkin lymphoma. Blood. 2006;108(2):662-668

30. Panico L, Tenneriello V, Ronconi F, et al. High CD20+ background cells predict a favorable outcome in classical Hodgkin lymphoma and antagonize CD68+ macrophages. Leuk Lymphoma. 2015;56(6) 1636-1642.

31. Sanchez-Espiridion B, Montalban C, Lopez A, et al. A molecular risk score based on 4 functional pathways for advanced classical Hodgkin lymphoma. Blood. 2010;116(8): e12-17.

32. Griss J, Bauer W, Wagner C, et al. B cells sustain inflammation and predict response to immune checkpoint blockade in human melanoma. Nat Commun. 2019;10(1):4186.

33. Cader FZ, Schackmann RCJ, Hu X, et al. Mass cytometry of Hodgkin lymphoma reveals a CD4(+) regulatory $\mathrm{T}$-cell-rich and exhausted T-effector microenvironment. Blood. 2018;132(8):825-836.

34. Carey CD, Gusenleitner D, Lipschitz M, et al. Topological analysis reveals a PD-L1associated microenvironmental niche for Reed-Sternberg cells in Hodgkin lymphoma. Blood. 2017;130(22):2420-2430. 